\title{
Uma Abordagem para Melhoria Contínua do Processo de Desenvolvimento de Software
}

\author{
Viviane Malheiros ${ }^{1,2}$, Fábio Rilston Paim², Henrique Guzzo², Manoel G. de \\ Mendonça Neto ${ }^{1}$ \\ ${ }^{1}$ NUPERC - Universidade Salvador (Unifacs) \\ Salvador - BA - Brasil \\ ${ }^{2}$ Serviço Federal de Processamento de Dados (SERPRO) \\ São Paulo - SP - Brasil \\ \{viviane.malheiros, fabio-rilston.paim, henrique-luiz.guzzo\} \\ aserpro.gov.br, mgmneunifacs.br
}

\begin{abstract}
Designing software processes for large enterprises is a hard task, but evolving this process can be even harder. This article describes an approach to address typical issues regarding organizational standard software process improvement and to foster continuous evolution of it. We present GM-PSDS, a process improvement support tool that manages the improvement proposals flow. GM-PSDS is being used at Brazil's Federal Data Processing Service(Serpro), a very large software organization. Its facilities are described having the organizational process that supports them as a backbone. We discuss how GM-PSDS contributes to a more effective process improvement, based on analyses elaborated upon the tool corporate database.
\end{abstract}

Resumo. Processos de software para grandes empresas são reconhecidos como de difícil definição. Todavia, o processo de evolução contínua que sucede sua implantação pode ainda exceder em complexidade a etapa inicial de definição. Este artigo descreve a abordagem do SERPRO para superar gargalos inerentes a essa evolução e assegurar a melhoria contínua de seu processo padrão, baseada no uso de uma ferramenta para a gestão do fluxo de propostas de melhoria. A ferramenta é apresentada tendo-se os processos organizacionais que lhes dão suporte como pano de fundo, enquanto discutese a contribuição da ferramenta para a melhoria efetiva do processo, por meio de análises extraídas a partir de sua base de dados.

\section{Introdução}

Diversos estudos relacionam a qualidade do software à qualidade do processo que o gera [Humphrey, 1990; Fiorini, 2001; IEEE, 2001; SEI, 1993; Pádua, 2003]. Bons processos devem ajudar a produzir: melhor; mais barato; mais rápido [Pádua, 2003]. Quando inexiste um processo definido, ou ainda quando este existe, mas não é utilizado, as chances de o software não atender às expectativas de prazo, custo ou qualidade tornamse altas. McFeeley [1996] defende que a melhoria de processos de software é um desafio para as organizações. Para ser considerada madura pelo SEI (Software Enginnering 
Institute) [SEI, 2002], uma organização necessita, além de definir e utilizar um processo de software, melhorá-lo continuamente. $\mathrm{O}$ estabelecimento de um processo padrão para a organização e a gestão de um programa de melhoria do processo estão previstos no nível 3 do CMMI (Capability Maturity Model Integration), por meio das áreas de processo: Organizational Process Definition (OPD) e Organizational Process Focus (OPF). Ambas, em especial a área de foco no processo, reúnem práticas relacionadas à melhoria contínua do processo organizacional. Essas práticas destacam a necessidade de compreensão e tratamento dos pontos fortes e oportunidades de melhoria identificadas para o processo.

A melhoria contínua do processo pode se provar, muitas vezes, mais complicada e sujeita a falhas do que a definição primária de um processo padrão. O desafio que se coloca é identificar procedimentos que possam ser estabelecidos e automatizados, de forma a promover o tratamento efetivo das melhorias propostas, revertendo-as em otimização do processo, após criteriosa análise das mesmas.

Este artigo apresenta a ferramenta GM-PSDS (Gestão de Mudanças do Processo SERPRO de Desenvolvimento de Soluções), desenvolvida pela empresa SERPRO (Serviço Federal de Processamento de Dados) [SERPRO,2006] para suporte à melhoria contínua do seu processo padrão. Da forma como foi estruturada, a solução apresentada pode ser aplicada em outras empresas com apenas pequenos ajustes, relacionados com a estrutura da organização (fluxo do tratamento de melhorias e suporte).

A ferramenta viabiliza a análise, o controle e a monitoração de oportunidades de melhoria, vinculando-as aos ganhos obtidos para o processo, numa abordagem de trabalho cooperativo. O desenvolvimento da ferramenta está apoiado num extenso programa de melhoria do processo de alcance corporativo, conforme descrito na Seção 2. A Seção 3 apresenta os processos definidos para tratamento de oportunidades de melhoria, cuja automação é descrita na Seção 4. Em seguida, apresentamos os resultados da aplicação da ferramenta no SERPRO e suas contribuições para a melhoria controlada do processo.

\section{Melhoria do Processo Organizacional}

O SERPRO implantou o PSDS - Processo SERPRO de Desenvolvimento de Soluções [Paim, Tavares, 2002; Malheiros, Mendonça Neto, 2005a], seguindo uma necessidade crescente em modernas empresas de software de melhorar o seu processo de desenvolvimento. O objetivo do PSDS é fornecer um processo de software padronizado e dinâmico às suas equipes de desenvolvimento, que permita extrair as melhores práticas de desenvolvimento de software da competência individual dos membros de equipes de projeto, para serem avaliadas e disponibilizadas para utilização por todas as áreas [Malheiros, Mendonça Neto, Farias, 2002].

O PSDS oferece a base para responder às perguntas: "quem faz o que", "quando" e "como", no contexto dos ambientes organizacional e de processos. Sua estrutura está baseada em macro-atividades, um conjunto relacionado de atividades que podem ser referentes tanto a atividades de Engenharia de Software quanto de Gestão de Projetos [Malheiros, 2002]. Concebido como uma ferramenta Web, o site do PSDS é livremente inspirado em elementos do RUP (Rational Unified Process [Kruchten, 1999; RUP, 2005]) e do PMBoK (Project Management Body of Knowledge [PMI, 2004]) adaptados 
às melhores práticas definidas nos modelos CMM (Capability Maturity Model) e CMMI.

Como um software, o PSDS está em evolução contínua e possui procedimentos definidos para incorporação de melhorias ao processo, que iniciam com uma Proposta de Melhoria ao Processo (PMP).

\subsection{Programa de Melhoria do Processo}

Um programa de âmbito organizacional denominado Programa SERPRO de Melhoria do Desenvolvimento de Soluções (PSMDS) foi criado para prover a institucionalização do PSDS [Malheiros, 2005b]. Este programa visa garantir treinamento, recursos e fundos para incorporação do PSDS pelas unidades de desenvolvimento, e mantê-lo alinhado com as boas práticas de mercado. O programa trata o processo de desenvolvimento de forma corporativa, auferindo-lhe a independência necessária à formulação das políticas e procedimentos e à padronização das práticas executadas pelas unidades do SERPRO que desenvolvem software.

As diretrizes estabelecidas para esse programa foram: (a) manter e evoluir o PSDS, tendo como referências os modelos CMM e CMMI; (b) monitorar o uso do PSDS pelas unidades diretamente envolvidas com o desenvolvimento de soluções de software; (c) mapear aspectos de simplicidade e praticidade na definição dos procedimentos e padrões do PSDS, visando a melhoria da qualidade das soluções desenvolvidas; (d) fortalecer grupos de qualidade de software como indutores do uso adequado do processo; (e) buscar continuamente a melhoria no nível de atendimento aos clientes; (f) definir requisitos de ferramentas de apoio ao processo; e (g) buscar a integração com outros processos e projetos empresariais. A Figura 1 mostra os papéis organizacionais definidos para viabilizar essas metas.

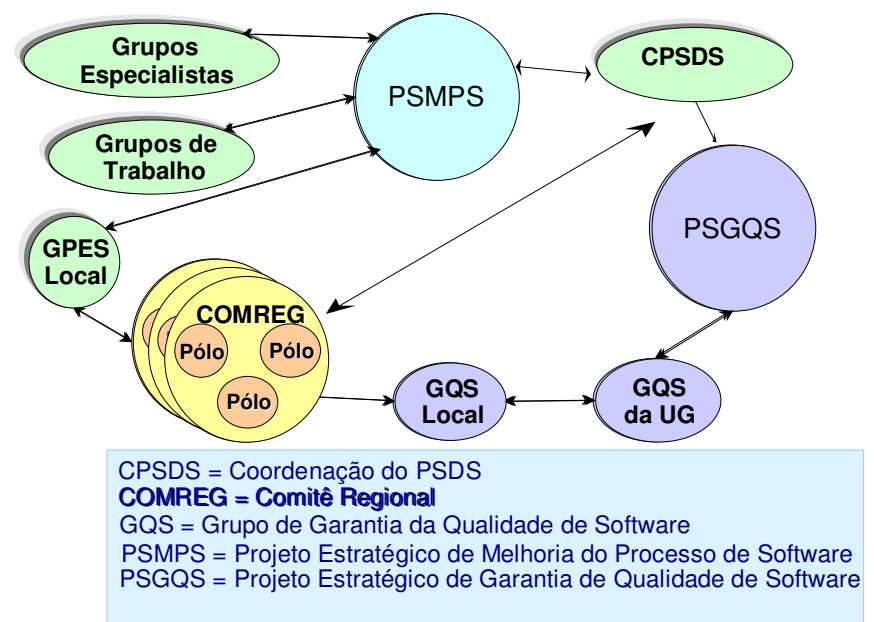

Figura 1. Estrutura para melhoria do processo de desenvolvimento

Os grupos Coordenação do PSDS (CPSDS), Projeto Estratégico de Melhoria do Processo (PSMPS) e Garantia da Qualidade de Software Corporativa (PSGQS) fazem parte da estrutura formal da empresa e são diretamente responsáveis pela execução do programa. Os demais grupos são formados por colaboradores de diversas áreas de desenvolvimento: Grupos Especialistas (GE), mantenedores dos ativos do processo; Grupos de Processo Locais (GPES Local), guardiães do processo nas unidades; 
Comitês Regionais de Processo (COMREG), e ouvidores regionais. Envolver diretamente colaboradores de áreas de desenvolvimento na manutenção do processo (GE) e institucionalização do processo nas unidades (GPES Local e COMREG) traz como benefício a incorporação da vivência e da competência individual dos membros das equipes para o processo.

O PSMPS, equivalente ao process group do CMMI [SEI, 2002], é o grupo responsável por facilitar a definição, manutenção e melhoria do processo utilizado pela organização para o desenvolvimento de soluções.

A melhoria contínua do processo de desenvolvimento é feita através de Propostas de Melhoria do Processo (PMP), que são tratadas seguindo-se um fluxo colaborativo de análise que permeia o GPES Local, PSMPS e os GEs. À medida em que o processo vai sendo utilizado pelas unidades de desenvolvimento e vai sendo implantado em novas unidades, oportunidades de melhoria são identificadas e propostas pelos desenvolvedores. Os grupos GQS e COMREG também contribuem para a melhoria de processo ao identificar e submeter PMPs para análise. Um consultor de GQS, por exemplo, no seu contato com as equipes de desenvolvimento, pode identificar lacunas no processo que estão favorecendo não conformidades recorrentes. Para esses casos, ele criará PMPs na ferramenta GM-PSDS. Todo o ciclo de vida de uma PMP desde sua geração até sua aprovação ou rejeição é acompanhado pela ferramenta GMPSDS.

\subsection{Gestão de Processo da Organização}

A Gestão do Processo da Organização (GPO) é a macro-atividade do PSDS que regulamenta e descreve os procedimentos para a definição, manutenção e customização do próprio processo. Ela também estabelece atividades de melhoria contínua da estrutura e conteúdo do PSDS, de forma que este esteja sempre atualizado e ajustado à realidade das diversas áreas de desenvolvimento da empresa. Essa macro-atividade utiliza as práticas preconizadas no CMMI, nas áreas de processo OPF e OPD, para a definição de práticas corporativas próprias de gestão do PSDS.

As atividades previstas para a macro-atividade Gestão do Processo da Organização (GPO) são:

(a)Avaliar o uso do processo de software;

(b)Gerenciar propostas de melhoria do processo;

(c)Planejar a evolução do processo;

(d)Evoluir o processo da organização;

(e)Controlar a geração de releases;

(f)Acompanhar a evolução do processo;

(g)Prover suporte ao processo;

\section{Ferramenta de Apoio à Melhoria do Processo Organizacional}

A ferramenta GM-PSDS é uma aplicação desenvolvida sobre a plataforma $A R S$ Remedy [BMC, 2006] para atender à sistemática de Gestão de Mudanças e Suporte ao 
PSDS definida na macro-atividade GPO - Gestão do Processo da Organização. Ela se constitui na única porta de entrada para registro e tratamento de Propostas de Melhoria do Processo (PMP) e, adicionalmente, de Pedidos de Suporte ao Processo (PS).

A ferramenta pode ser utilizada de duas formas:

a) Perfil Corporativo - Esta opção está disponível apenas aos integrantes dos grupos PSMPS, GPES Locais e GE (na modalidade cliente/servidor), responsáveis pelo tratamento de PMPs e PSs, desde o seu cadastro, encaminhamento e registro de pareceres até a sua aprovação ou rejeição, no caso de PMP, ou solução no caso de PS.

b) Perfil Usuário - Esta opção está disponível a todos os usuários do PSDS, acionando-se botão existente na parte superior do site do PSDS. Através desta facilidade, podem ser registrados e consultados PMPs e PSs. Esta modalidade utiliza a interface Web do ARS-Remedy e, portanto, não requer instalação de aplicativos ou componentes na estação do usuário.

A ferramenta GM-PSDS apóia o PSDS na automatização das atividades "Gerenciar Propostas de Melhoria do Processo" e "Prover Suporte ao Processo" da macro-atividade de GPO, gerenciando todo o ciclo de vida das PMPs e PSs. O Quadro 1 mostra as sub-atividades apoiadas diretamente pela ferramenta. Indiretamente a ferramenta fornece subsídios para a execução das atividades: Controlar a geração de releases e Acompanhar a Evolução do Processo.

\section{Quadro 1.Sub-atividades apoiadas diretamente pela GM-PSDS}

\begin{tabular}{|c|c|c|}
\hline \multicolumn{3}{|c|}{ Atividade: Gerenciar Propostas de Melhoria do Processo (PMP) } \\
\hline & Sub-atividade no PSDS & Ação na ferramenta \\
\hline 1 & Elaborar proposta de melhoria & Registro da PMP/PS \\
\hline 2 & $\begin{array}{l}\text { Efetuar análise preliminar e } \\
\text { Enviar proposta para análise }\end{array}$ & Registro do parecer do GPES Local \\
\hline 4 & Analisar proposta de melhoria & Colocação da PMP em análise pelo PSMPS \\
\hline 5 & Solicitar parecer de grupo especialista & Registro da solicitação de parecer \\
\hline 6 & Elaborar parecer & Registro do parecer pelo GE \\
\hline 7 & Aprovar proposta de melhoria & Registro da aprovação/rejeição da proposta \\
\hline \multicolumn{3}{|c|}{ Atividade: Prover Suporte ao Processo } \\
\hline & Sub-atividade no PSDS & Ação na ferramenta \\
\hline 1 & Registrar pedido de suporte & Registro da PMP ou PS \\
\hline $\begin{array}{l}2 \\
3\end{array}$ & $\begin{array}{l}\text { Realizar atendimento em primeiro nível e } \\
\text { Encaminhar ao suporte de segundo nível }\end{array}$ & $\begin{array}{l}\text { Registro do parecer do GPES Local (solução ou } \\
\text { encaminhamento ao PSMPS) }\end{array}$ \\
\hline 4 & Solicitar parecer de grupo especialista & Registro da solicitação de parecer \\
\hline
\end{tabular}




\begin{tabular}{|c|c|c|}
\hline 5 & Elaborar parecer & Registro do parecer pelo GE \\
\hline 6 & Realizar atendimento em segundo nível & Registro da solução do Pedido de Suporte \\
\hline 7 & Gerar PMP & --- \\
\hline 8 & Cadastrar Solução Global & --- \\
\hline
\end{tabular}

Através da ferramenta GM-PSDS, todos os dados relativos à melhoria do processo são armazenados de forma relacional e podem ser acessados através de consultas SQL pré-definidas ou customizadas pelo usuário.

A interface principal da ferramenta é mostrada na Figura 2. Ela oferece recursos ao usuário final para encontrar respostas a perguntas como: (a) Qual a situação atual de uma PMP? (b) Para quem a PMP foi enviada? (c) Qual o mecanismo de envio (correio eletrônico, malote, telefone)? (d) A PMP já foi analisada? (e) Por quem ela foi analisada? (f) Quando ela foi aberta? (g) Há quanto tempo permanece sem solução? (h) Quantas PMP foram abertas, aprovadas, implantadas, por regional e por UG?

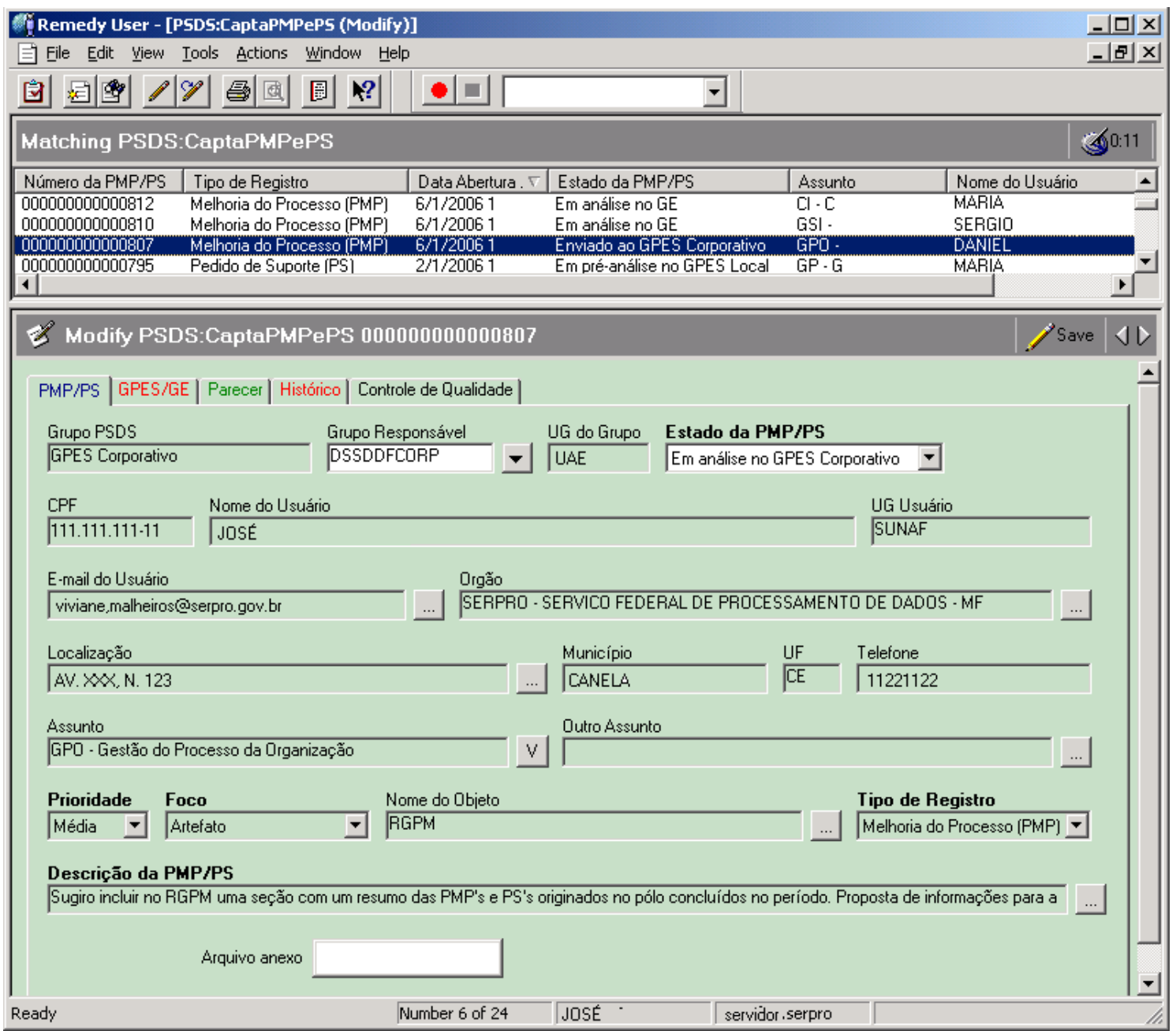

Figura 2. Extrato da interface principal da GM-PSDS, mostrando detalhes de PMP 


\section{Aplicação da Ferramenta no SERPRO}

A GM-PSDS está em uso no SERPRO desde novembro de 2004. Após mais de um ano, já é possível avaliar a sua real contribuição para a análise e acompanhamento das propostas de melhoria ao PSDS. A utilização da ferramenta trouxe ganhos sensíveis na administração da evolução do PSDS. Toda PMP só é reconhecida pelo PSMPS quando registrada na ferramenta. Assim, nenhuma alteração do processo pode ser processada sem que haja PMP associada e aprovada, que a justifique. Essa prática tem o efeito colateral positivo de facilitar o controle da configuração do PSDS.

O suporte ao uso do PSDS (Pedido de Suporte - PS), entretanto, devido a sua necessidade de rápido atendimento, não se processa exclusivamente através da ferramenta, sendo provido de diversas outras maneiras, como, por exemplo: consultoria, mentoring; suporte informal realizado pessoalmente ou por telefone. Essas formas de suporte são bastante úteis, ágeis e produtivas. Mas, para que, ao menos, os pedidos de suporte mais significativos possam ser compartilhados com todos os usuários e para que seja possível, por meio da reincidência destes pedidos, identificar pontos de melhoria para o PSDS, os especialistas prestadores de suporte são orientados a registrar a realização do atendimento na ferramenta GM-PSDS. Adicionalmente a inclusão de um pedido de suporte à base da GM-PSDS poderá viabilizar sua futura incorporação à base de conhecimento.

O acompanhamento dos tratamentos de PMP e PS é feito semanalmente, quando é gerado um relatório para cada supervisor de GE com todas as submissões pendentes. Adicionalmente, uma vez por mês, é gerado um relatório para o coordenador PSMPS (projeto de melhoria do processo) com o consolidado mensal do tratamento das PMPs e PSs. Para analisar a evolução do processo de melhoria contínua, semestralmente são geradas medições que viabilizam a análise da sistemática de melhoria como um todo. $\mathrm{Na}$ última coleta, foram selecionados para análise: todas as PMPs, pareceres e PSs registrados na base de novembro de 2004 a janeiro de 2006 e todas as avaliações de atendimento (Controle de Qualidade) solicitadas ou registradas no período.

A quantidade de PMP e PS abertos no primeiro ano de utilização da ferramenta GM-PSDS reflete um alto grau de comprometimento dos usuários com a melhoria do processo. Foram submetidas 820 solicitações, média de mais de três submissões por dia útil. Desse total, $79 \%$ foram PMPs (651) e 21\% foram PSs (169).

Das 651 PMPs tratadas, $72 \%$ foram aprovadas para implantação no PSDS e $28 \%$ foram rejeitadas. $\mathrm{O}$ alto percentual de aprovação das propostas reflete a pertinência e a qualidade das submissões.

A Figura 3 mostra o percentual de PMPs atendidas por macro-atividades. A quantidade de PMP finalizadas (aprovadas ou rejeitadas) corresponde a 63\% do total. Esse foi o mesmo índice de finalização obtido no último levantamento semestral. Apesar de esse número ser um reflexo da incidência de PMPs de alta complexidade, a meta estabelecida para a próxima medição é de $70 \%$. Esta meta é mostrada como uma linha horizontal na Figura 3. 
A Figura 4 mostra o percentual de PSs atendidas por macro-atividades. O atendimento a PS está sendo mais conclusivo, $75 \%$ dos PSs foram solucionadas rapidamente e apenas $25 \%$ estão aguardando uma solução. Para o PSDS, o atendimento aos PSs é prioritário, já que a solução rápida de um PS pode agregar qualidade ao produto final. A prioridade dada a esse atendimento no segundo semestre de 2005 se refletiu na melhora do índice de finalização dos PSs, que subiu de $63 \%$ para $75 \%$. A meta esperada para a próxima medição é de $80 \%$. Esta meta é mostrada como uma linha horizontal na Figura 4. Os dados das duas figuras foram obtidos durante o mesmo período de tempo. Analisando as duas figuras é possível perceber que o atendimento a Pedidos de Suporte foi priorizado por todas as macro-atividades.

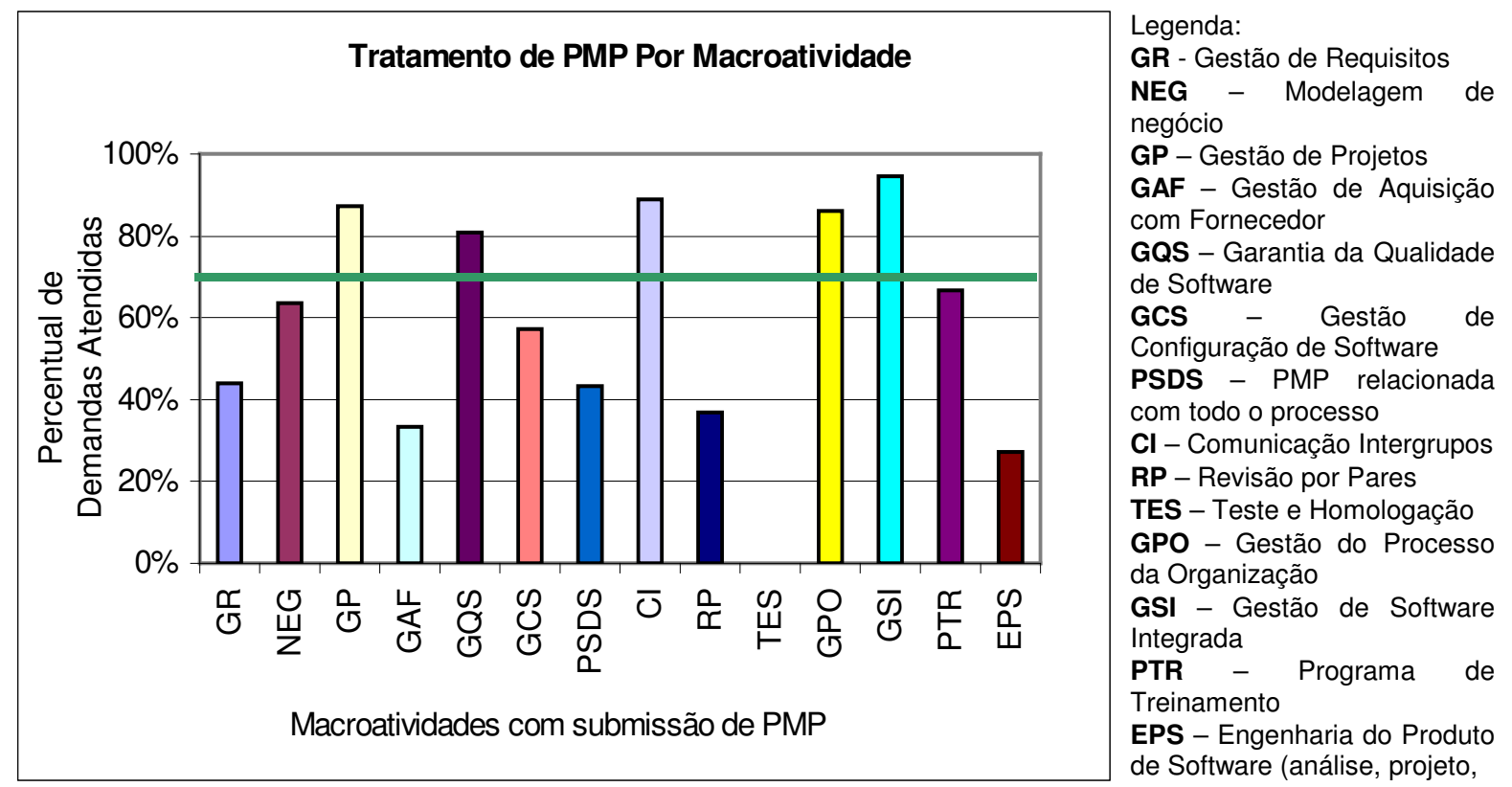

Figura 3. Finalização no tratamento de PMPs por macro-atividade

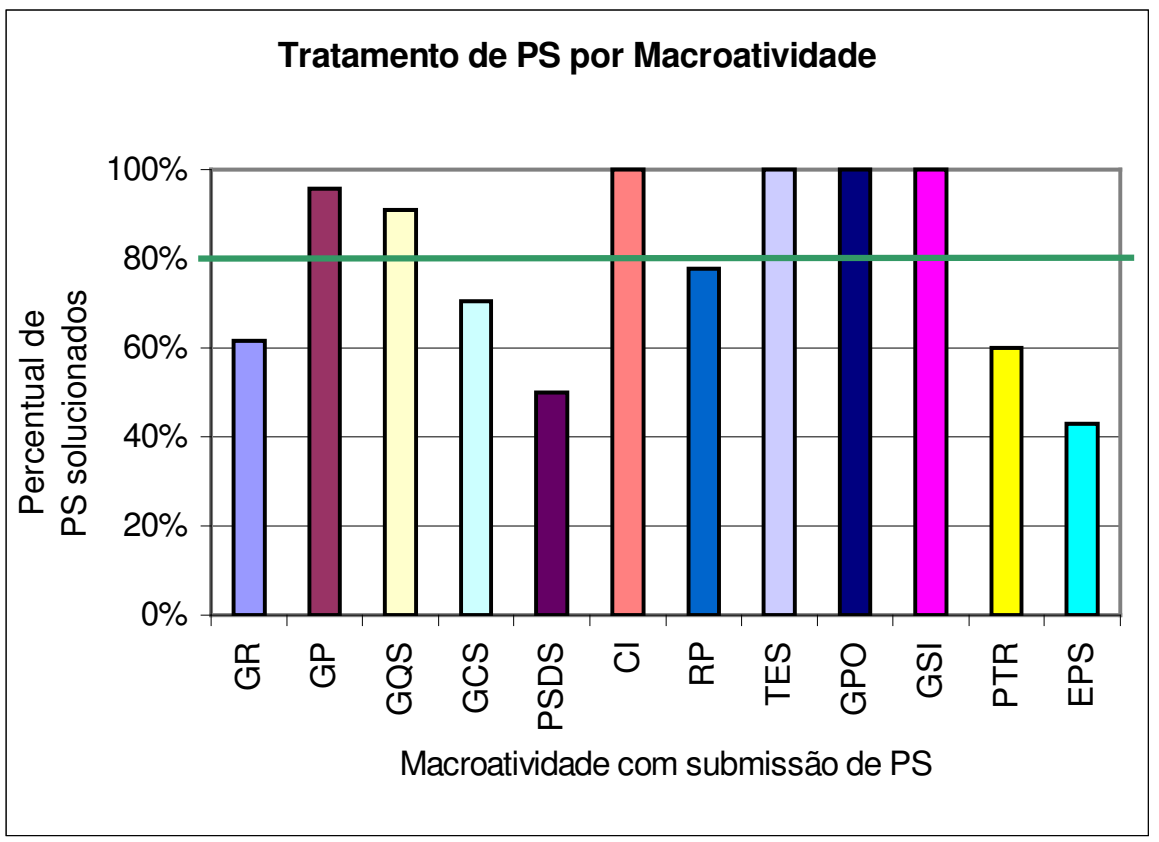

Legenda:

GR - Gestão de Requisitos GP - Gestão de Projetos

GQS - Garantia da Qualidade de Software

GCS - Gestão de Configuração de Software

PSDS - PMP relacionada com todo o processo

Cl - Comunicação Intergrupos

RP - Revisão por Pares

TES - Teste e Homologação

GPO - Gestão do Processo da

Organização

GSI - Gestão de Software Integrada

PTR - Programa de

Treinamento

EPS - Engenharia do Produto de Software (análise, projeto, implementação, implantação )

Figura 4. Finalização no tratamento de PMPs por macro-atividade 
As Figuras 3 e 4 permitem identificar quais os grupos que estão mais perto ou já atingiram as metas. Dessa forma, é possível a tomada de ações direcionada para os grupos que ainda estão distantes das metas. Os grupos que mais se destacaram na análise de OS geralmente são os grupos que mais se destacaram no atendimento de pedidos de suporte. A única exceção é o grupo de Testes.

Algumas questões que ainda precisam ser tratadas para garantir o tratamento rápido dos PSs, e aumentar o seu encaminhamento através da ferramenta GM-PSDS são:

(a) Nem sempre o tempo destinado às atividades de análise de PMP é suficiente. Se por um lado, um grupo de especialistas formado por equipes de desenvolvimento tem a vantagem de trazer a visão de quem pratica o processo para a análise de melhorias, por outro gera problemas de prioridade na execução das atividades relacionadas ao processo.

(b) Alguns grupos especialistas não conseguem envolver rapidamente todos os seus membros, que são formados por desenvolvedores que atendem ao cliente e possuem um gerente funcional diferente do gerente coordenador do grupo especialista.

A Figura 5 ilustra que, atualmente, as macro-atividades relacionadas com o CMMI nível 3 são responsáveis por $44 \%$ das submissões de PMPs à ferramenta, enquanto que as macro-atividades relacionadas com o nível 2 são responsáveis por $56 \%$ das submissões. Considerando a quantidade de unidades que utilizam as macroatividades do nível 3 (4 unidades das 43 unidades de desenvolvimento), esse número pode parecer elevado. O PSMPS atribui esse percentual elevado de PMPs relacionados com o nível 3 a dois motivos: (1) os processos de nível 3 são mais novos e portanto estão mais suscetíveis a propostas de melhoria; (2) as unidades que já estão executando as macro-atividades do nível 3 são as unidades que utilizam o PSDS a mais tempo (mais de 3 anos) e estão mais conscientes da importância da melhoria do processo.

Um outro indício de que a valorização da melhoria contínua do processo é diretamente proporcional à maturidade da equipe é que a unidade de negócio que mais contribuiu para a melhoria do processo foi a que possui mais unidades qualificadas internamente no CMM nível 2 com 55\% das submissões.

A partir da distribuição de PMPs por macro-atividade é possível identificar as macroatividades do processo que precisam de revisão e os grupos de trabalho que estão mais sobrecarregados. É possível também inferir, com alguma precisão, as macro-atividades que estão sendo utilizadas mais fortemente pelas equipes.

O foco das submissões ao GM-PSDS está prioritariamente relacionado com as atividades, sub-atividades e procedimentos do PSDS (39\%), seguido pelos artefatos do PSDS (37\%) e pelas ferramentas utilizadas no desenvolvimento (14\%). Os outros $10 \%$ das submissões não foram classificadas em nenhum dos três focos.

Em relação ao tipo de mudança, 58\% das submissões foram classificadas como manutenções evolutivas, corretivas ou adaptativas. Desse total, $24 \%$ foram classificadas como manutenção corretiva. Esse número (cerca de $13 \%$ das propostas estarem relacionadas com algum tipo de correção) é considerado aceitável pelo PSMPS, que, 


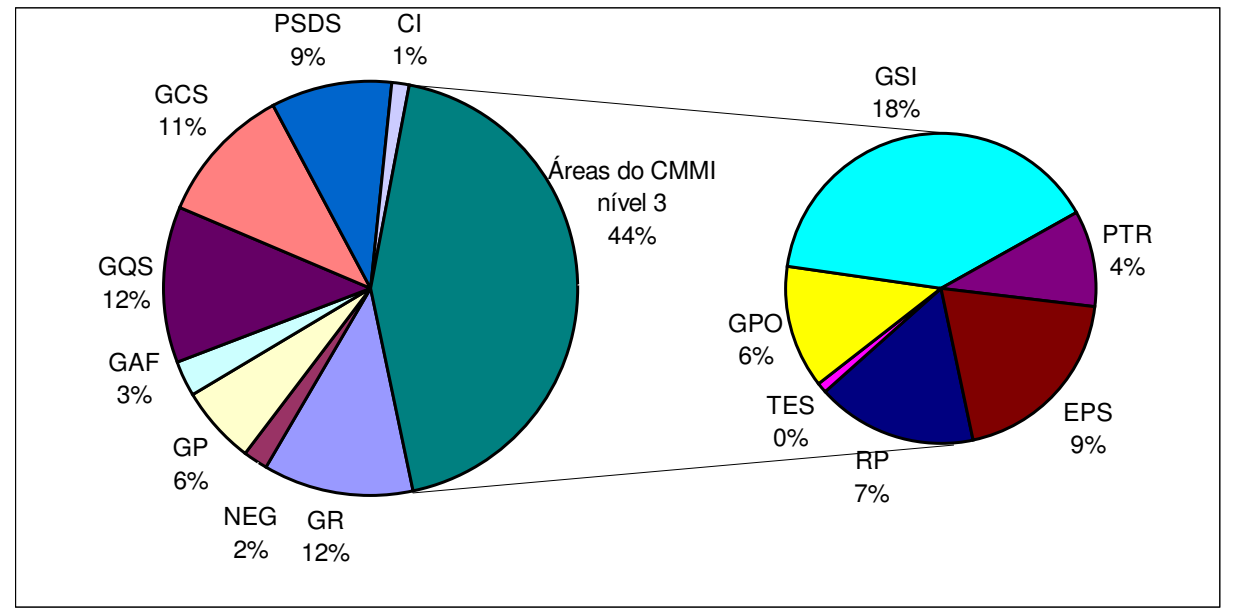

Legenda:

GR - Gestão de Requisitos, NEG - Modelagem de negócio, GP - Gestão de Projetos,

GAF - Gestão de Aquisição com Fornecedor, GQS - Garantia da Qualidade de Software,

GCS - Gestão de Configuração de Software, PSDS - PMP relacionada com todo o processo,

Cl - Comunicação Intergrupos, RP - Revisão por Pares, TES - Teste e Homologação,

GPO - Gestão do Processo da Organização, GSI - Gestão de Software Integrada ,

PTR - Programa de Treinamento ,

EPS - Enaenharia do Produto de Software (análise. proieto. imnlementacão. imnlantacão )

ainda assim, atuará junto aos GEs para intensificar os testes e as homologações do processo.

Seguindo uma abordagem similar ao que é proposto por Falbo [Falbo, 1998] e Villela [Villela, 2004], a GM-PSDS possui um recurso para assinalar uma PMP para inclusão na Base de Conhecimentos ou na FAQ (Perguntas Freqüentes) do PSDS. Há indícios, no entanto, que este recurso não está sendo utilizado na sua plenitude. Apenas quatro submissões $(0,5 \%$ do total) foram marcadas para inclusão na Base de Conhecimentos e apenas cinco (0,6\% do total) foram marcadas para inclusão na FAQ. Esses indicativos são importantes para a melhoria da Gestão do Conhecimento na organização. Os baixos índices de submissão obtidos até agora apontam para a necessidade de reforçar a importância desses indicativos para os grupos especialistas.

As macro-atividades de Gestão de Requisitos com 47 PSs, Gestão de Configuração de Software com 27 PSs, e Gestão de Projetos com 23 PSs, são as que mais demandaram pedidos de suporte. Isto pode revelar a necessidade de treinamento suplementar, ou melhorias na própria macro-atividade, para facilitar sua utilização. Revela também o uso intenso dessas macro-atividade pelos desenvolvedores.

Os locais que mais demandaram suporte são: Recife (práticas de nível 3), São Paulo e Rio de Janeiro, resultado esperado já que São Paulo e Rio de Janeiro têm a maior concentração de desenvolvedores.

Do total de itens submetidos, $61 \%$ foram analisados localmente, em um primeiro nível de suporte, minimizando ou subsidiando o trabalho de análise do grupo corporativo. Esse número indica um alto nível de conhecimento já disseminado nos grupos de desenvolvimento e demostra uma grande distribuição do trabalho. 
Foram ao todo solicitados 680 pareceres para os grupos especialistas (GE). Isso significa que $83 \%$ das solicitações foram avaliadas, não só pelo grupo corporativo, mas também pelos colaboradores das unidades que trabalham no desenvolvimento de software e se especializam em uma das macro-atividades do PSDS.

Os grupos especialistas (GE) mais solicitados na emissão de pareceres foram: Gestão de Requisitos com 199 pareceres (17,5\%), Gestão de Software Integrada com 112 pareceres $(16,4 \%)$ e Garantia da Qualidade de Software com 105 pareceres $(15,4 \%)$. Estes três grupos são responsáveis por metade dos pareceres solicitados.

O nível de satisfação com o atendimento das PMPs e PSs pode ser considerado muito bom. A satisfação quanto à resposta dada à solicitação é maior do que a satisfação quanto ao prazo decorrido para esta resposta, o que revela que os grupos estão bem qualificados para atender às demandas. Mas revela também que o PSMPS, os GPES Locais e os GEs podem ser mais ágeis no atendimento das solicitações. A nota dada aos prazos definidos para a implementação de PMP aprovadas é um pouco menor. A justificativa é que a implantação da melhoria só estará disponível na maioria dos casos no próximo release do PSDS. A decisão de empacotar diversas alterações num único release, visa dar um tratamento mais adequado à evolução do PSDS. As implantações são feitas em releases bem controlados para não impactar seus usuários com muitas versões de processo.

\section{Conclusão e trabalhos futuros}

A ferramenta GM-PSDS tem se mostrado um instrumento efetivo para a execução e acompanhamento da melhoria contínua do processo de desenvolvimento. Através da ferramenta é possível gerir propostas de melhoria ao processo de desenvolvimento e pedidos de suporte. É possível também responder a perguntas como: (a) Qual a situação atual de uma PMP? (b) Para quem a PMP foi enviada? (c) Qual o mecanismo de envio (correio eletrônico, malote, telefone)? (d) A PMP já foi analisada? (e) Por quem ela foi analisada? (f) Quando ela foi aberta? (g) Há quanto tempo permanece sem solução? (h) Quantas PMP foram abertas, aprovadas, implantadas, por regional e por UG?

Questões relativas à gestão de conhecimento também podem ser analisadas a partir dos dados registrados na ferramenta, como que áreas precisam de mais treinamento, quem detém o conhecimento sobre determinado assunto, que áreas geram mais dúvidas, entre outras questões relevantes ao assunto.

A partir de análise dos dados registrados pela ferramenta é possível estabelecer metas e acompanhar se as mesmas foram alcançadas, de forma a garantir a melhoria contínua do processo, inclusive do processo de gestão do PSDS. Além disto, alguns dos dados coletados, através da ferramenta, e sua análise podem ser de grande valia para a comunidade acadêmica de engenharia de software e para outras empresas que estejam trabalhando com melhoria de processo ou implantação do CMMI. Através dos dados coletados é possível identificar, por exemplo, as áreas de processo que geraram mais oportunidades de melhoria.

Além da análise dos dados, a estrutura organizacional montada para a melhoria de processo e a própria ferramenta de gestão de mudanças no processo são importantes contribuições para a academia e para empresas que estejam adotando práticas de 
melhoria contínua de processos, especialmente aquelas que utilizam o modelo CMMI como referência.

Algumas oportunidades de trabalhos futuros já foram identificadas, a partir da análise realizada:

a) o desenvolvimento de funcionalidades para acompanhamento da implementação e implantação de uma melhoria no PSDS, após a sua aprovação. Atualmente o último estado de uma PMP é "aprovada". A criação dos estados "em implementação", "implementada" e "implantada" pode ser muito útil;

b) A análise mais detalhada da base de dados gerada pela GM-PSDS, através de técnicas de mineração de dados para a geração de novo conhecimento a respeito da melhoria contínua do PSDS. A base conta hoje com 820 submissões de PMPs e PSs, além de 680 pareceres sobre os PMP. A análise manual de dados de quem detém determinado conhecimento ou qual o tipo de erro recorrente em relação a artefatos do PSDS está sendo realizada, mas demanda um tempo considerável. Identificar padrões de oportunidades de melhoria pode ser muito útil. A mineração visual de dados também pode ser muito interessante para a identificação de redes de conhecimento e gestão de competências da empresa.

c) O benchmarking entre unidades e com outras empresas para verificar se o perfil de demandas de melhoria contínua do processo do SERPRO é o mesmo que o de outras organizações; e a partir daí, fazer novos direcionamentos e reavalia as metas estabelecidas.

d) Analisar o tempo médio necessário para solucionar as propostas de melhoria e pedidos de suporte

\section{Referências}

BMC, (2006). ARS-Remedy: http://www.remedy.com/solutions/servicemgmt/itsm.html, acessado em 01-Março-2006.

Falbo, Ricardo de A. (1998) "Integração de Conhecimento em um Ambiente de Desenvolvimento de Software". Rio de Janeiro. Tese de Doutorado, COPPE/ UFRJ.

Fiorini, S. "Arquitetura para Reutilização de Processos de Software (2001). Tese (Doutorado) - Informática, Pontifícia Universidade Católica do Rio de Janeiro, 2001.

Humphrey, Watts S. (1990) "Managing the Software Process". Addison-Wesley Publishing, Company, Massachussets.

IEEE Software Engineering Coordinating Committee (2001) "SWEBOK: Guide to the Engineering Body of Knowledge - Trial Version 1.00" . Disponível em: http://www.swebok.org. Acesso em junho 2004.

Kruchten, P. (1999). The Rational Unified Process. Addison-Wesley, USA.

McFeeley, R. "IDEAL: A User's Guide for Software Process Improvement"(1996) CMU/SEI-96-HB-001, ADA305472. Pittsburgh, PA: Software Engineering Institute, Carnegie Mellon University. Disponível em: http://www.sei.cmu.edu/publications/documents/96.reports/96.hb.001.html 
Malheiros, V., Mendonça Neto,M., Farias, L. (2002) "Uma abordagem de Gerência de Projetos de Software, sob o enfoque da Gestão do Conhecimento", In: Segunda Jornada Ibero-americana de Engenharia de Software e Engenharia de Conhecimento, Salvador. Anais da JIISIC 2002, v.1. p.1 - 6 .

Malheiros, V., Mendonça Neto,M. “Análise do tratamento de riscos em projetos de

desenvolvimento de software de uma organização" (2005a). In: $19^{\circ}$ Simpósio Brasileiro de Engenharia de Software, Uberlândia- MG. Anais.

Malheiros, V. "Metodologia para Avaliação da Gerência de Riscos em Empresas de Desenvolvimento de Software" (2005b). 199 f. Dissertação (Mestrado em Redes de Computadores), Universidade Salvador, Salvador.

Pádua, W., Paula, F. (2003) "Engenharia de Software: Fundamentos, Métodos e Padrões". Editora LTC. ISBN: 8521613393.

Paim, F. R. S., Tavares, H. C. (2002). "Implementing a Living Software Process". In: Proceedings of the First ICSE Workshop on Software Quality (WoSQ), Orlando, Flórida, USA (Maio).

PMI - Project Management Institute (2004) “A Guide to the Project Management Body of Knowledge (PMBOK Guide)". Project Management Institute. Disponível em: http://www.pmi.org/ Acesso em julho 2005.

Pressman, Roger S. (2004) "Engenharia de Software". Editora TECMEDD. ISBN: 8586804258.

RUP - IBM Rational Unified Process. "Rational Unified Process". Disponível em: http://www-306.ibm.com/software/awdtools/rup/ . Acesso em julho 2005.

SEI - Software Engineering Institute. "Key Practices of the Capability Maturity Model" versão 1.1. CMU/SEI-93-TR-025. Fevereiro, 1993. Disponível em: http://www.sei.cmu.edu/cmm/ . Acesso em junho 2005.

SEI - Software Engineering Institute. "Capability Maturity Model Integration versão 1.1" (2002). Disponível em: http://www.sei.cmu.edu/cmm/. Acesso em junho 2005.

SERPRO - Serviço Federal de Processamento de Dados. Disponível em: http://www.serpro.gov.br. Acesso: janeiro 2006.

Villela, K. "Definição e Construção de Ambientes de Desenvolvimento de Software Orientados a Organização" (2004). Tese (Doutorado) - Universidade Federal do Rio de Janeiro UFRJ. 